\title{
Development of Person Localization and Activities Recognition System
}

\author{
Liandi Gao, Shuaib Tao, Changqing Ji, Baofeng Wang and Zumin Wang* \\ College of Information Engineering \\ Dalian University \\ Dalian, China \\ wangzumin@163.com; 943799867@qq.com
}

\begin{abstract}
This paper first presents the trends and related works of localization and activity recognition of human body. Then we introduced the discussion of multiple studies for person localization and activities recognition. And finally we can see that the ceiling binary infrared sensor network for locating and recognition showed growing advantages which were not limited by light condition, obstacles, fast computing, and most of all, this system can preserve individual privacy to some extent. Moreover, this system is with high precision, stable performance, high reliability, low cost and low power consumption. So it solves some current existing problems in human behavior recognition system.
\end{abstract}

Keywords-infrared sensors; localization; activities recognition; binary values

\section{INTRODUCTION}

Recently, with the rapid development of sensor network and to provide multiple personalized services in users' daily life, human body's localization and activities recognition have received extensive discussion and attention. Some video cameras have been used in the research of person localization. [1] tried to use cameras for targets' localization. However, regardless of the young and the old, they would feel uncomfortable under the observing of the camera for a long time in daily life. Moreover, individual privacy can not be preserved effectively and camera's vision is largely limited by light condition and obstacles. In that way the traditional research method digital image/video processing is not suitable for localization and activities recognition in home environment. Schulz et al. [2] attempted to use an ID badge for person localization and authentication. However, there are so many people, especially the old, would be uncomfortable and unwilling to wear such sensing devices. Then [3-4] tried to use pyroelectric infrared sensors for human identification and localization in a relatively small room. At the same time, the first try about ceiling sensor system is applicable for a large room [5].

And with the coming aged tendency of population in our country, the elderly who lives alone may fall at home but nobody knows. Further, if they cannot call for help, this issue may turns even worse. It's very dangerous if he or she does not receive timely assistance. Thus it is also very indispensable to detect abnormal activities of the elders.

Generally, there are many kinds methods of human body activities recognition, such as recognition based on vision (camera), based on wearable sensor devices, based on smartphone, based on infrared sensor etc.

In a word, infrared sensing system is superior to the others. Because the system based on infrared sensor network is gradually showing the advantages of privacy protection, no disturbance in daily life etc. And the detection areas of the infrared sensors installed on the ceiling can cover the whole room. In that way there will be no dead zone of detection.

In [6] ,the renovated infrared sensing system, sampling rate of $80 \mathrm{~Hz}$ for up to 128 nodes using $250 \mathrm{kbps}$ equilibrium line has been achieved. [6] proposed a novel method for person localization to bring a finer precision $(31.6 \mathrm{~cm})$ than that of the geometrical precision $(1.5 \mathrm{~m})$ of sensor placement.

\section{RELATED WORKS}

There are many kinds of researches about human behavior analysis. [7] used wearable inertial sensors for human daily activities recognition. Rasheed used kinematic sensors based on smartphone with alarm notification service [8]. User has to attached smartphone with different body parts of himself. [9] applied the change of signal intensity of $\mathrm{WiFi}$ to analyze person's behavior. However, the recognition rate was influenced by external factor, such as if the ambient WiFi signal is very weak and unstable, the accuracy of recognition will be very low. Vision-based human action recognition is the process of labeling image sequences with action labels. Image representations and the subsequent classification process are discussed separately to focus on the novelties of recent research in [10]. In [11], it had introduced an overview about Human Behaviour Analysis (HBA) for Ambient-Assisted Living (AAL) and aging in place purposes focusing especially on vision techniques. [12] the field of views (FOVs) of the PIR sensors is modulated by two degrees of freedom (DOF) of spatial segmentation. The localization algorithm is proposed to fuse the data stream generated from different sensor nodes within the wireless network. The Kalman Filter and Kalman Smoother are utilized to refine the estimation of the human position.

These systems [1], [10], [13] using cameras can exactly obtain high accuracy of recognition under an enough light condition, but in indoor environment, such as at home or at office, little lower recognition rate does not cause a serious problem. Furthermore, it cannot protect individual privacy and may cause uncomfortable about users. The general comparison 
between infrared sensor module and camera devices are shown in Table I.

TABLE I. COMPARISON BetweEn CAMERAS AND SENSING DeVices

\begin{tabular}{c|c|c}
\hline & $\begin{array}{c}\text { Infrared sensor } \\
\text { module }\end{array}$ & Camera \\
\hline Place to use & Anywhere indoor & Indoor and outdoor \\
\hline $\begin{array}{c}\text { Recognition } \\
\text { accuracy }\end{array}$ & Lower & Higher \\
\hline Privacy protection & Strong & Weak \\
\hline Light condition & No special condition & Stable light \\
\hline Limitation about & No limit & limited \\
Obstacle condition & & High and fixed \\
\hline Installation cost & Low and flexible & \\
\hline
\end{tabular}

In order to overcome the effects of obstacles, light condition, tackle with complex data and preserve individual privacy, [14] use a binary ceiling infrared sensor network for multiperson locating and soft tracking. Soft tracking means little physical or psychological disturbance with users. On the contrary, hard tracking means undermine user's privacy.

Compare with the research of wearable acceleration sensing devices, it has no need any cooperation from user. In addition, it has the advantages of small amount of data to deal with, and the speed for computing is very fast, and it can be free from light condition.

In [15], twenty infrared sensors were evenly attached to the ceiling in simulated home environment. We took the $4 * 5$ sensor network as a "top view camera" with a very poor resolution: 20 pixels with binary values/levels. To increase the gray level, we took a short-duration average of observed binary values at each pixel. The novelty of this study is that we use BP neural network to recognize different activities that share similar area, i.e., the areas are overlapping when the target does different activities. In this paper, on the basis of those "pixel values" and BP neural network, seven activities are recognized.

In addition, such a method will be further applied to detect abnormal behavior of a single elderly person, such as fall detection of old citizens. As there are more and more elderly people who are living independent, it is essential to concern and monitor their behavio at home. If the elderly falls down at home with no conscious and no in time assistance, this will aggravate this bad condition, and may be cuase a disease,such as senile dementia, even worse, may be dead. So it is significant to set up a stable system to pay attention to the movements of the old.

\section{INFRARED SENSOR SYSTEM}

\section{A. Infrared sensor}

"Pyroelectric infrared sensors", sometimes is also called "infrared motion sensors", are attached to the ceiling in home environment for human body behavior analysis and tracking [16]. The infrared sensor can detect the change of temperature in the surrounding caused by the movement of a target. The detection area of ceiling infrared sensor, which projects to the floor is a circle with a certain diameter. The detection range of infrared sensor module is shown in Table II. In the system of [16], there is a hand-made cylindrical lens hood, which is used for sensors' detection area of magnification and minimization.

TABLE II. DETECTION PERFormance OF INFRARED SENSOR MODULE

\begin{tabular}{|l|c|}
\hline Detection distance & $5 \mathrm{~m}$ \\
\hline Horizontal detection range & $100^{\circ}$ \\
\hline Vertical detection range & $80^{\circ}$ \\
\hline Detection area & $64 \mathrm{zones}$ \\
\hline Movement speed & $0.5 \mathrm{~m} / \mathrm{s}(\mathrm{min})-1.5 \mathrm{~m} / \mathrm{s}(\mathrm{max})$ \\
\hline target & $\mathrm{H} 700 \mathrm{~mm} \times \mathrm{W} 250 \mathrm{~mm}$ \\
\hline
\end{tabular}



Fig. 1. The side view of infrared sensor

[15] used AMN3111 sensor which contained 16 lenses that were used for collecting infrared light from the four quadrant of the surface of the infrared sensor. Each sensor module obtains a binary value response at each time frame.The infrared sensor array has the following characteristics: the moving target may activate multiple sensors in the same time. So the sensor outputs 1 and in the same time LED will be lightened. On the contrary, when a target is in a stationary or with slight movement, the sensor module is inactive, i.e., the sensor outputs 0 and LED cannot be lightened. Moreover, we can adjust a desirable sampling rate through software program. The side view of infrared sensor is as shown in Fig. 1 and the interconnection of twenty sensors in ceiling is shown in Fig.2. And the top view layout of infrared sensor array is as shown in Fig. 3. 


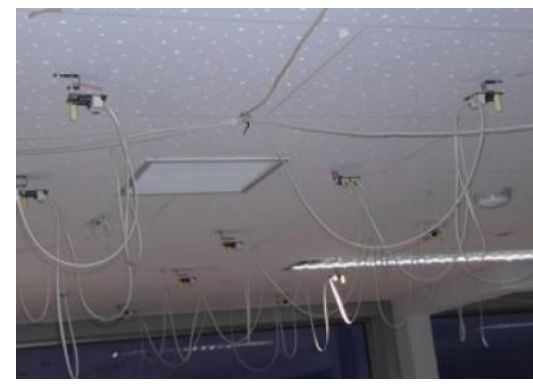

Fig. 2. The interconnection of infrared sensor array

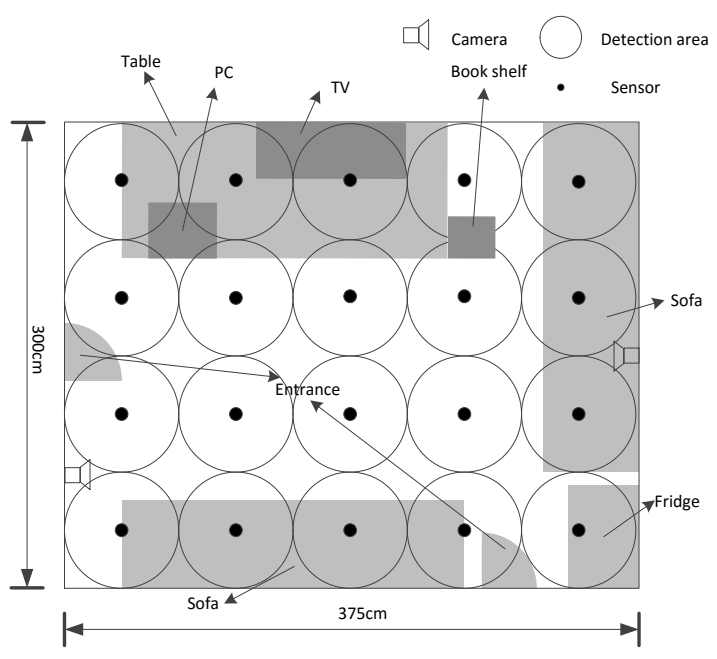

Fig. 3. The top view layout of infrared sensor array

\section{B. Pixel Value}

Based on the installation and layout characteristics of this infrared sensor system [17], we can take it as a low resolution top view camera with 20 pixels and low gray level with two levels. Similarly, we take sensors' responses as the top view image with 0 or 1 gray level. In order to increase the gray level of the pixel, we take the weighted average of the binary values at each pixel. So the gray level is spreading over $[0,1]$. By using the distribution of the pixel points at different gray levels, the binary values can be accumulated to locate a target's position over a short period of time. The optimization method of the binary value is proposed in [17]. And the gray level at time $t$ is as shown in Fig. 4.

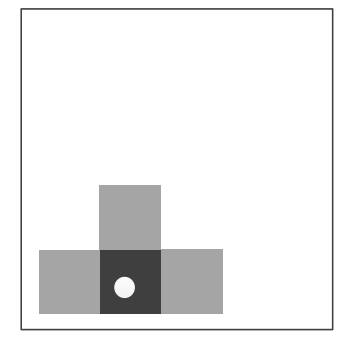

Fig. 4. The binary gray level image

By accumulating the binary values in a short time, the scope of the expression of active sensors has been extended. The spatial resolution about the moving target is enhanced by taking the weighted average of the positions information of active sensors.

\section{Person Localization}

For detecting the abnormal behavior of users, [18] has attached some kinds of sensors to doors, kitchenware (washing machine and microwave) in indoor environment. However, this system cannot offer the current position and trajectory of user. Moreover, such a system is very hard to be mounted. Because there are many obstacles in such an environment.

In order to be free from obstacles and increase the sampling rate and avoid the noise about infrared sensors, [17] has improved the system by the use of binary infrared sensors mounted to ceiling. There are two methods to localize target's position, i.e., linear and nonlinear methods. Both methods can achieve the localization of person. Experimental result has showed that the nonlinear method achieved a better performance. We can get a short-term trajectory of the person in [16].

\section{Activities Recognition}

In the study of [15], we have proposed BP neural network for target activities recognition. BP neural network is a kind of multi-layer feed forward network, which is trained by error back propagation algorithm. It is one of the most widely used neural network models. Its learning rule is to use the steepest descent method. Through the reverse propagation to constantly adjust the weights and thresholds of the network, the sum of squared error of the network can reach the minimum.

A target will do different activities in different time and in similar region. We can recognize different activities effectively which roughly share the same region by BP neural network classification algorithm, e.g. walking and sweeping, both share similar regions, the classifier may be confused. But if take the speed of different activities into account, we can distinguish them roughly. In the experiment, two cameras are mounted to the ceiling in simulated home environment, which are for recording the true activities of target. That is, the recordings provide references for the BP neural network algorithm to recognize different activities for classifying.

In the environment of neural network toolbox of MATLAB, $\mathrm{BP}$ neural network reads binary values stored in txt format. We choose seven kinds of sample sets from the data collected by four experimenters. In each kind of sample set, there are 300 groups of data samples for training and 100 groups of data samples for testing. Setting appropriate training function, the training steps, minimum error and network of target output. Then we used 10-fold cross-validation about input samples. When error reaches a predetermined requirement or training reaches a preset learning steps, it's time to stop training. Finally, the average recognition rate is calculated by the 10 -fold crossvalidation. The final recognition rate we get is $84.7 \%$. This rate is not as high as camera, but it is relatively high with such low resolution.

Experimental result showed that this system can recognize different behavior in similar area. And the recognition accuracy is considerable.

\section{DISCUSSION}

In this system [15], the ceiling binary infrared sensor network is to recognize different activities of single target in simulated home environment. It preserves individual privacy 
and with little disturbance of user' s daily life. The noise has been avoided by using the binary values of infrared sensors. That made the amount of computation has reduced, the speed of operation has improved. In addition, infrared sensors are not subject to the constraints of light and obstacles. While camera observing results can be effected by them all. In this paper, the detection range of infrared sensors covers the whole simulated home environment, i.e., there is no detection of dead zone. Meanwhile, this recognition system is low-cost and easy to install.

However, there are some limitations. That is, in this system, the recognition rate is not as accurate as the traditional camera. The recognition rate even will be reduced if there many activities largely share associated areas. Rather, there is no need very high accuracy recognition of person at home or in office. So, it is applicable to use ceiling binary infrared sensor network to localize the position of human body and recognize different activities.

\section{CONCLUSION}

In this paper [15], we propose a ceiling binary infrared sensor system to recognize different activities. The optimization method based on the binary values is for locating the moving target. By using BP neural network classification algorithm, multiple activities conducted by targets can be recognized effectively. Through experiments, we can get such a conclusion, the behavior recognition rate in the system is $84.7 \%$ and is $4.7 \%$ higher than the sensors fixed in home appliance. The ceiling binary infrared sensor can detect multiple targets (in this paper there is only one single moving target in the simulated home in each experiment)in future. The infrared sensor network system preserves individual privacy and reduces disturbance to user's daily life, etc. This paper provides the basis for the next study of the elderly abnormal behavior recognition and fall detection, as well as multipersons' identifying and tracking.

\section{ACKNOWLEDGMENT}

This work is supported by the National Natural Science Foundation of China (Grant No. 61501076 and 61370199), Dalian Key Laboratory of Smart Medical and Health, the general program of Liaoning Provincial Department of Education Scientific Research (L2014492 and L2014283), Guangdong Provincial Key Laboratory of Petrochemical Equipment Fault Diagnosis (Open-ended fund no. GDUPTKLAB201505).

\section{REFERENCES}

[1] T. Y. Lee, T. Y. Lin, S. H. Huang, S. H. Lai, and S. C. Hung, "People localization in a camera network combining background subtraction and scene-aware human detection," International Conference on Advances in Multimedia Modeling, Springer-Verlag, Vol. 6523, pp. 151-160, 2011.
[2] Schulz D, Fox D, Hightower J, "People tracking with anonymous and IDsensors using Rao-Blackwellised particle filters," International Joint Conference on Artificial Intelligence. Morgan Kaufmann Publishers Inc, pp. 921-926, 2010.

[3] Shankar M, Burchett J B, Hao Q, "Human-tracking systems using pyroelectric infrared detectors," Optical Engineering, vol. 45, pp.106401, 2006.

[4] Z. Zhang, X. Gao, J. Biswas, "Moving targets detection and localization in passive infrared sensor networks," International Conference on Information Fusion. pp.1-6, 2007.

[5] Hosokawa T, Kudo M, Nonaka H, "Soft authentication using an infrared ceiling sensor network," Formal Pattern Analysis \& Applications, vol. 12, pp. 237-249, 2009.

[6] Nonaka H, Tao S, Toyama J, et al, "Ceiling sensor network for soft authentication and person tracking using equilibrium line," Peccs 2011 Proceedings of the, International Conference on Pervasive and Embedded Computing and Communication Systems, Vilamoura, Algarve, Portugal, pp. 218-223, 5-7 March, 2011

[7] M. Zhang and A. A. Sawchuk, "Human daily activity recognition with sparse representation using wearable sensors," Biomedical \& Health Informatics IEEE Journal of, vol. 17, pp. 553-560, 2013.

[8] M. B. Rasheed, N. Javaid, and T. A. Alghamdi , "Evaluation of human activity recognition and fall detection using android phone," IEEE, International Conference on Advanced Information NETWORKING and Applications. pp. 163-170, 2015.

[9] Y. Gu, L. H. Quan, M. N. Chen, F. J. Ren, "Research on passive human activity recognition using WiFi ambient signals," vol. 4, pp. 308-313, 2015.

[10] Poppe R, “A survey on vision-based human action recognition,” Image \& Vision Computing, vol. 28, pp. 976-990, 2010.

[11] A. A. Chaaraoui, P. Climent-Pérez, F. Flórez-Revuelta, "A review on vision techniques applied to human behaviour analysis for ambientassisted living," Expert Systems with Applications, vol. 39, pp. 1087310888, 2012.

[12] Luo X, Liu T, Shen B, "Human indoor localization based on ceiling mounted PIR sensor nodes," IEEE Consumer Communications \& NETWORKING Conference. IEEE, pp. 868-874, 2016.

[13] W. Y. Lin, Y. Z. Chen, J. X. Wu, H. L. Wang, B. Sheng, and H. X. Li, “ “ A new network-based algorithm for human activity recognition in videos, " Circuits and Systems for Video Technology, IEEE Transactions on, vol. 24 , pp. 826-841, 2014.

[14] S. Tao, M. Kudo, B. N. Pei, "Multiperson locating and their soft tracking in a binary infrared sensor network," IEEE Transactions on HumanMachine Systems, vol. 45, pp. 550-561, 2015.

[15] L. D. Gao, S. Tao, C. Q. Ji, B. F. Wang, Z. M. Wang, "Design for Human Body Activities Recognition System Based on Ceiling Binary Infrared Sensor Network," unpublished.

[16] S. Tao, M. Kudo, and H. Nonaka, “Privacy-preserved behavior analysis and fall detection by an infrared ceiling sensor network," Sensors, vol. 12, pp. 16920-16936, 2012.

[17] S. Tao, M. Kudo, H. Nonaka, "Recording the activities of daily living based on person localization using an infrared ceiling sensor network," IEEE International Conference on Granular Computing. IEEE, pp. 647 652, 2011.

[18] Sawai K, and Yoshida M, "Algorithm to detect abnormal states of elderly persons for home monitoring," Systems \& Computers in Japan, vol. 38, pp. 34-42, 2007. 